\title{
Stochastic User Behaviour Modelling and Network Simulation for Resource Management in Cooperating Mobile Telecommunications and Broadcast Networks
}

\author{
Angelo Centonza, Gareth A. Taylor, Takebumi \\ Itagaki, Thomas J. Owens, John Cosmas and \\ Yong-Hua Song
}

School of Engineering and Design, Brunel University, Kingston Lane, Uxbridge, Middlesex, UB8 3PH, United Kingdom E-mail: Angelo.Centonza@brunel.ac.uk, Gareth.Taylor@Brunel.ac.uk, T.Itagaki@Brunel.ac.uk, Thomas.Owens@brunel.ac.uk, John.Cosmas@brunel.ac.uk, Y.H.Song@brunel.ac.uk

\begin{abstract}
The latest generations of telecommunications networks have been designed to deliver higher data rates than widely used second generation telecommunications networks, providing flexible communication capabilities that can deliver high quality video images. However, these new generations of telecommunications networks are interference limited, impairing their performance in cases of heavy traffic and high usage. This limits the services offered by a telecommunications network operator to those that the operator is confident their network can meet the demand for. One way to lift this constraint would be for the mobile telecommunications network operator to obtain the cooperation of a broadcast network operator so that during periods when the demand for the service is too high for the telecommunications network to meet, the service can be transferred to the broadcast network. In the United Kingdom the most recent telecommunications networks on the market are third generation UMTS networks while the terrestrial digital broadcast networks are DVB-T networks. This paper proposes a way for UMTS network operators to forecast the traffic associated with high demand services intended to be deployed on the UMTS network and when demand requires to transfer it to a cooperating DVB-T network. The paper aims to justify to UMTS network operators the use of a DVB-T network as a support for a UMTS network by clearly showing how using a DVB-T network to support it can increase the revenue generated by their network.
\end{abstract}

Keywords: Traffic forecast, user behaviour, hybrid network, mobile service, network simulation.

Reference to this paper should be made as follows: Centonza, A., Taylor, G.A., Itagaki, T., Owens, T.J., Cosmas, J., Song, Y.-H., (200-) 'Stochastic User Behaviour Modelling and Network Simulation for Resource Management in Cooperating Mobile Telecommunications and Broadcast Networks', International Journal of Mobile Communications, ----.

Biographical notes: Angelo Centonza obtained his BSc and MSc with 


\section{A. Centonza, G.A. Taylor, T. Itagaki, T.J. Owens, J. Cosmas and Y.-H. Song}

honours in July 2002 from Politecnico di Bari, Italy, where he was extensively involved in the research areas of Power Electronics and Signal Processing. Currently, he is a doctoral research student in the area of Hybrid Networks at the Department of Electronics and Computer Engineering of Brunel University, West London.

Gareth A. Taylor received his BSc in 1987, MSc in 1992 and PhD in 1997 from the School of Computing and Mathematical Sciences, University of Greenwich, UK. Currently he is a lecturer in network design and computer systems at the Department of Electronic and Computer Engineering of Brunel University, West London.

Takebumi Itagaky obtained a BEng degree in Applied Physics at Waseda University in Tokyo, Japan, a Postgraduate Diploma in Music Information Technology at City University in London and a PhD at University of Durham. In 1998 he was appointed as a lecturer at Glasgow Caledonian University, thence in 2000 as a lecturer at Brunel University.

Thomas J. Owens obtained his $\mathrm{PhD}$ in Electrical and Electronic Engineering from Strathclyde University in 1986. In 1987 he joined the Department of Electronic and Computer Engineering, Brunel University as a lecturer. He is a Chartered Engineer, Chartered Mathematician, and a member of the Institute of Teaching and Learning. He is also the author of more than 60 refereed journal and conference publications.

John Cosmas obtained his $\mathrm{PhD}$ in Image Processing and Pattern Recognition at Imperial College, University of London in 1987. Between 1978 and 1983, he worked as an electronics development engineer at Tube Investments and Fairchild Camera and Instruments. In 1983 he joined Imperial College, University of London as a Research Student and in 1986 Queen Mary \& Westfield College, University of London as a Lecturer in Digital Systems Design and Telecommunications. In 1999 he joined Brunel University as a Reader in Multimedia Systems. He is currently Professor of Multimedia Systems at Brunel University. His research interests mainly concern video processing and Multimedia Systems.

Yong-Hua Song obtained his BEng, MSc, and PhD in 1984, 1987 and 1989, respectively. He joined Brunel University in 1997 as Professor of Network systems at the Department of Electronic and Computer Engineering. Currently he is director of Brunel Research Institute of Network Systems. He has published four books and over 300 papers concerning the applications of intelligent and heuristic computational methods in engineering systems. 
Stochastic User Behaviour Modelling and Network Simulation for Resource

Management in Cooperating Mobile Telecommunications and Broadcast Networks

\section{Introduction}

This paper is based on research carried out as part of the "Converged IP based Services for Mobile Users and Networks in DVB-T and UMTS Systems" (CISMUNDUS) project [1] that was a European Union-funded research and development project investigating the convergence of digital broadcast and mobile telecommunications.

In major European economies such as those of Germany, France, Italy and the UK, digital broadcasting is supplying broadcasters with new audiences by virtue of the fact that the digital broadcast signal can be received on portable and mobile terminal devices. In the UK the standard adopted for digital broadcasting is the Digital Video Broadcasting Terrestrial (DVB-T), which can deliver high-resolution video services with coverage up to nationwide areas. Although the current implementation of DVB-T does not provide the built-in return channel essential for interactive TV and on demand services, a return channel can be made available using mobile telecommunications networks. The use of such a return channel would result in the possibility of deploying new on-demand multimedia services for both mobile telecommunications and broadcast network users, generating increased revenue for the broadcasters and a net "add on" income for the telecommunications operators. Mobile telecommunications companies are in fact looking for value added services to offer to their subscribers while at the same time trying to minimize additional infrastructure investment. They are aware of the fact their users are ready to consume new multimedia services provided that they are delivered with satisfactory quality of service [2], [3]. Broadcast offers a cost efficient solution for distributing multimedia content to a large audience. Thus, the mobile telecommunications network operators can potentially benefit from the possibility of delivering their services through cooperating telecommunication and broadcast networks. The CISMUNDUS project investigated and developed prospective services intended for a mobile terminal device with access to a network consisting of a DVB-T network cooperating with a Universal Mobile Telecommunication Systems (UMTS) network. The different prospective services designed within the CISMUNDUS project are all intended to be managed in such a way that when the service demand reaches a certain value, the service delivery is handed over from the telecommunications network to the broadcast network. The reason for this is that the prospective services described in subsequent sections are highly resource demanding and as such would easily cause congestion if delivered to a large number of users on a UMTS network. On the other side, the deployment of such services in situations of low demand could be successfully handled by telecommunications networks and could generate for their operators an increase in revenue. Switching the delivery of a service from one network to the other when a certain service demand is reached could allow the exploitation of the advantages of IP services delivered over mobile telecommunications networks as well as the benefit 
of the point to multipoint communication available with broadcasting, generating an increase in revenue for both broadcast and telecommunications operators with minimum infrastructure investment. This is the reason why a solution for service delivery involving both broadcast and telecommunications networks was proposed in the CISMUNDUS project.

In the past, partial solutions to the problem of highly resource demanding service delivery have been proposed such as the design of new methods for radio resource allocation as described in [4] and [5] were new mechanisms for optimising the usage of the capacity available in third generation mobile networks are proposed. However, these methods are not designed to satisfy massive numbers of requests for new multimedia services. Such services could easily consume all the available capacity and therefore result in a breach of the service level agreements as contracted with the users. In the CISMUNDUS project it was proposed that the delivery of highly resource demanding services should be controlled by a unit called the Content Delivery System (CDS) that would decide whether to deliver the service on the broadcast network or on the telecommunications network. This decision could be made on the basis of resource monitoring and the number of service subscriptions. Such a service deployment mechanism would have to be configured by setting thresholds for the maximum numbers of users accessing the services via UMTS. When the number of subscribers for a particular service is lower than the threshold the deployment would be done using UMTS, otherwise the service would be delivered using DVB-T. However, some services may be so resource demanding that a "one way" deployment via "DVB-T only" is the best solution for the current infrastructure. The design of such a resource management system requires estimation of the traffic that would be generated by the services that are planned for deployment on the cooperating networks. The approach taken in this paper is to design stochastic models from which a forecast of the traffic generated by these prospective services can be gained. This solution could provide network operators with important information as to whether or not the prospective services could be successfully deployed on the telecommunications network alone or whether at times of high demand it would be necessary to transfer the service to a support network such as a broadcast network. Similar types of traffic and user behaviour models can be found in the literature and have been applied to the most commonly used wireless applications. In [6] and [7] for example, models for temporal and spatial distributions of traffic in urban areas and for traffic forecasting have been developed for GSM networks. However, the main service considered in these models is the circuit-switched voice service, which makes the modelling of the traffic load relatively simple due to the one-to-one relationship between users and channels. A similar research path has been followed in [8], where the authors again propose models describing the traffic on both a temporal and spatial basis but also consider services such as wireless Internet. Therefore, the models are closer to an actual third generation services scenario but do not provide a forecast of the tremendous increase of traffic that could be generated by the deployment of multimedia applications such as video on demand. In addition, statistical models at both the packet and session level can be found in [9], [10], [11] and [12]. In such cases source traffic can be 
Stochastic User Behaviour Modelling and Network Simulation for Resource

Management in Cooperating Mobile Telecommunications and Broadcast Networks

represented by stochastic models that can give a prediction of what the traffic load would be for a specific application under certain conditions. Again, the mathematical models do not consider the consequences that the deployment of new interactive multimedia services could have on the traffic generated and therefore on the overall network performance.

In this paper the problem of what the traffic load could be due to the deployment of new multimedia applications is addressed. Attempts of solving such problem have already been carried out in the past [13], but the results achieved did not rely on real data. The user behaviour models hereby presented are based on data collected from Internet servers that provided similar services to those being analysed. Although the models derived are based on analysis performed within a particular service scenario, the concepts behind their design could be applied to any service scenario using any network technology. This makes the proposed technique flexible and applicable to different cases. This paper does not address the problem of how different tariffs could influence traffic trends and this issue is left for future work. However, the models presented in section 4 and 5 were adopted to generate simulations using the OPNET simulation software [14]. Such simulation package is particularly suitable for network simulations and ahs already been adopted in the past [15]. The simulations demonstrate how third generation mobile networks could react to the deployment of highly requested multimedia services. These results provide telecommunications network operators with an estimate of the maximum service demand sustainable by their network, thereby giving an indication of what the service demand thresholds for the hand over of service delivery to a support broadcast network could be. Moreover, from the results obtained it can be seen how advantageous it could be to provide a mobile telecommunications network with a supporting broadcast network such as DVB-T. In such situations new routes could be available for the traffic during busy time periods to relieve the congestion on the telecommunications network.

This paper is organized as follows: in Section 2 a technical description of the deployment of highly requested multimedia applications in UMTS networks is presented. In Section 3, the service scenario adopted for analysis in Sections 3 and 4 is illustrated. Sections 4 and 5 present and illustrate the stochastic models designed to forecast the number of subscriptions per service. Section 6 reports the scenario simulated using the proposed traffic models and the network behaviour shown in the simulations. The conclusions drawn are presented in Section 7.

\section{Theoretical Analysis}

The delivery of highly demanded applications on new generations of mobile networks often represents a problem for telecommunications network operators. Frequently, operators refuse to deliver on their networks "user-attractive" multimedia services that could cause situations of congestion and degradation of the offered QoS. In this section 
the technical reasons for such phenomena are analysed the latest generation of mobile telecommunications networks in use in Europe at the moment, UMTS.

UMTS is a recognised standard for third generation mobile networks in Europe and adopts Wideband Code Division Multiple Access (W-CDMA) technology as its air interface. This allows all users to use the same wideband frequency for transmission of information over the physical channels. The use of orthogonal codes [16] makes it possible to encode and decode the signal avoiding interference from other users and allows the use of variable bit rates depending on the service. However, in order to have successful decoding the signal to interference ratio (SIR) has to be higher than a certain threshold. When a large number of users try to connect at the same time, the total wideband interference experienced by a single user increases. Therefore, the transmission power of individual user equipment (UE) has to increase, which causes a further increase of interference and consequently transmission power. This phenomenon will eventually cause the UE to use maximum transmission power, making it unable to react against an extra increase in noise. The connection in this case will be very weak and subject to possible packet loss and in the worst-case disconnection. A similar problem can be encountered at the base station with regard to downlink communication, with the difference that the transmission power is increased due to the path losses and the signal fading (proportional to the distance of the UE from the base station). Moreover, UMTS capacity is affected by the bit rate in use at each terminal, this bit rate being dependent on the QoS required. All such parameters can be combined together as illustrated in [17], to give an estimation of the maximum capacity that one cell can handle, either in the uplink or the downlink. In order to achieve this result let us start with the uplink case and define the energy per user bit $E_{b}$ divided by noise spectral density $N_{0}$ :

$$
\left(E_{b} / N_{0}\right)_{j}=\frac{W}{v_{j} R_{j}} * \frac{P_{j}}{I_{\text {total }}-P_{j}}=(\text { Processing Gain })_{j} * \operatorname{SIR}_{\mathrm{j}}
$$

Where $W$ is the UMTS chip rate, with regard to user $j, P_{j}$ is the received signal power at the base station, $v_{j}$ is the activity factor, $R_{j}$ is the bit rate and $I_{\text {total }}$ is the total received wideband power including the thermal noise power in the base station. Rearranging for $P_{j}$ gives

$$
P_{j}=\frac{1}{1+\frac{W}{\left(E_{b} / N_{0}\right)_{j} * R_{j} * v_{j}}} I_{\text {total }}
$$

Let us define $L_{j}=P_{j} / I_{\text {total }}$ to be the load factor $L_{j}$ of one connection then from the above

$$
L_{j}=\frac{1}{1+\frac{W}{\left(E_{b} / N_{o}\right)_{j}^{*} R_{j}^{*} v_{j}}}
$$


Stochastic User Behaviour Modelling and Network Simulation for Resource Management in Cooperating Mobile Telecommunications and Broadcast Networks

The total received interference, excluding the thermal noise $I_{T h}$, can be written as the sum of the received powers from all $N$ users in the same cell

$$
I_{\text {total }}-I_{T h}=\sum_{j=1}^{N} P_{j}=\sum_{j=1}^{N} L_{j} * I_{\text {total }}
$$

The noise rise is defined as the ratio of the total received wideband power to the thermal noise power and using Equation (1) can be obtained as

$$
\text { NoiseRise }=\frac{I_{\text {total }}}{I_{T h}}=\frac{1}{1-\sum_{j=1}^{N} L_{j}}=\frac{1}{1-\eta_{U L}}
$$

where we have defined the load factor $\eta_{U L}=\sum_{j=1}^{N} L_{j}$.

It can be seen from Equation (2) that as $\eta_{U L}$ tends to unity, the corresponding noise rise tends to infinity. This means that as the number of users served increases the system tends to saturation or more precisely to its pole capacity. After the pole capacity is reached any further request will, either be rejected by the admission control algorithm (in case of throughput based admission control [17]), or if accepted will cause an overload of the cell with consequent corruption of communication for the users connected. Additionally, in the load factor the interference from the other cells should be taken into account by the ratio

$$
i=\frac{\text { Other cell interference }}{\text { Own cell interference }}
$$

Taking into account the interference from the other cells the uplink load factor can be written as

$$
\eta_{U L}=(1+i) * \sum_{j=1}^{N}\left[\frac{1}{1+\frac{W}{\left(E_{b} / N_{0}\right)_{j} * R_{j} * v_{j}}}\right]
$$

Following a similar argument as for the uplink, the downlink load factor $\eta_{D L}$ can be defined as 


$$
\eta_{D L}=\left[\left(1-\alpha_{j}\right)+i_{j}\right] * \sum_{j=1}^{N}\left[\frac{1}{1+\frac{W}{\left(E_{b} / N_{0}\right)_{j} * R_{j} * v_{j}}}\right]
$$

where $\alpha_{j}$ is the downlink orthogonality factor for user $j$ and $i_{j}$ is the "other cell interference over the own cell interference" for user $j$. The parameter $i_{j}$ is different for each user because it depends on the users position within the cell. The load factor can provide an estimate of how much the network is loaded by comparing it with its maximum value of one and a forecast of how many users can simultaneously use a particular service in certain interference conditions as explained in the following example.

Let us suppose we want to estimate how many users in a single cell can simultaneously download a video clip using a maximum bit rate of 144 Kbps. In such a scenario, an average value found by simulation for $E_{b} / N_{o}$ was $3.2 \mathrm{dBm}$. The other cell to own cell interference can be set to 0.67 and the average orthogonality factor can be chosen as 0.6 as suggested in [17]. In this scenario the maximum number of users that can be served at the same time can be obtained by substituting the above mentioned values of $R_{j}, E_{b} / N_{o}, i_{j}$ and $\alpha_{j}, j=1,2, \ldots, N$, into Equation (4) and calculating for how many users $\eta_{D L}$ would reach its maximum value of one. Hence, the maximum number of users is determined to be 13 . Any increase in subscriptions, will either cause connection rejection or a higher level of interference in the cell causing corruption of the received signals at the base station.

Consider a situation where all the users, of a UMTS telecommunications network, in a football stadium receive an announcement for a video service showing the scoring of a goal from another match. If in an interval of time of 60 seconds the majority of users wanted to subscribe and consume the service this could generate an overload that could be not handled by the UMTS network. This is one of the many service scenarios studied in the CISMUNDUS project and gives an idea of how crowds of users interested in the same service could be the source of congestion for third generation telecommunication networks. For this reason, the scenario of a telecommunications network such as UMTS supported by a broadcast network such as DVB-T is analysed. The reason for the use of a DVB-T network in support of the UMTS network is to provide support in situations of over subscription through its high capacity and the use of broadcast channels instead of unicast. In the next section, the scenario adopted as a case study in this paper is presented.

\section{The Football Match Scenario}

The scenario studied in this paper is that of a football stadium where 60,000 people are watching a football match. The total number of UEs in the stadium and provided with a 
Stochastic User Behaviour Modelling and Network Simulation for Resource

Management in Cooperating Mobile Telecommunications and Broadcast Networks

terminal able to receive both UMTS and DVB-T signals is assumed to be 6,000, that is, the penetration is $10 \%$. The co-operating UMTS and DVB-T networks provide the area of the football stadium with multimedia services concerning the football match the users are watching as well as general services about football. This scenario has been chosen because it represents a good example of how different and numerous users localised in a restricted area can request the same services. Nevertheless, the methodology presented can be applied to different scenarios that could easily occur in real life. The examples include, groups of tourists visiting an archaeological site and being provided with multimedia services describing the history of the area, and numerous users in a traffic jam trying to access multimedia services providing information about the road traffic. These real life scenarios have one characteristic in common, large crowds of users requesting the same application.

The prospective services for the football scenario have been designed in the CISMUNDUS project together with their announcement and subscription schemes [18, 19]. The most important of them are listed below:

1) Match Program - Provides web pages with pictures and information about the match users are watching, the teams playing, the latest events concerning the match and links to pages containing information on other matches in the premier league. This service is continuously available to users.

2) Goal Alert - Provides video clips of the most important events (goals scored, free kicks, bookings, etc.) occurring in other matches playing at the same time in different locations. The service is announced to the users as soon as the event occurs by sending to them an alert message; therefore a high number of subscriptions are expected shortly after the announcement, such as within 60 to 80 seconds after the announcement.

3) Multi-view Football - Provides streaming videos from different angles of the match users are watching. This service would be announced once, before the start of the match and is accessible by the users at any time during the match provided that a subscription to the service has been made after the announcement. Because it demands high resources it is delivered using the DVB-T network only. A light version of this service for delivery via the UMTS network could consist of pictures and information describing the most important events of the match announced when the events occur, hence a pattern of subscriptions similar to that of the Goal Alert scenario could be forecast in this case.

In order to estimate the utilization of these services and consequently determine if they could be deployed solely via UMTS or would at some point require transferring to another delivery platform (in the scenario considered, a DVB-T network), user behaviour models need to be developed. Usually such models would be based on historical data generated either by monitoring users access to certain applications or by running service consumers surveys of different kinds as illustrated in [20]. However, no historical data 


\section{A. Centonza, G.A. Taylor, T. Itagaki, T.J. Owens, J. Cosmas and Y.-H. Song}

exists because the use of a DVB-T network in support of a UMTS network is only a proposal at the moment. Therefore, a similar service scenario must be found where users are expected to behave similarly, using similar services on a similar network. Such a scenario has been identified by analysing the access of Internet users to the Web site of the France '98 Football World Cup. This Web site provided Internet users around the world with a wide range of information. Besides being able to access the current scores of the football matches in real time, fans could also access previous match results, player statistics, player biographies, team histories, information on the stadiums and a wide range of photos and sound clips from the matches and interviews with players and coaches. In the next section an analysis of the Internet log access traces collected from the France98 Web servers is presented.

\section{User Behaviour Modelling}

To develop a user behaviour model of the "football match" scenario the workload of the 1998 World Cup Web Site has been analysed. However, such an analysis could be performed for different scenarios by identifying Internet services similar to those being considered. For the scenario considered in this paper the data traces from 1998 World Cup Web Site have been used, which are available on [21], while further analysis of the workload of the site can be found in [22]. The measurements from this site were made over a three-month period. During this time the site received a total of 1.35 billion requests. The data set used in the workload characterization study reported on [21] is composed of the access logs collected from each of the servers used by the World Cup Web site. Each access log is in the Common Log Format [21]. For every request received by the Web server, the following information was stored:

- $\quad$ Remotehost: the (encoded) IP address of the client issuing the request.

- $\quad$ rfc931: the remote login name of the user.

- $\quad$ authuser: the username by which the users authenticated themselves.

- [date]: the date and time of the request.

- $\quad$ request: the request line exactly as it comes from the client.

- $\quad$ status: the HTTP response status code returned to the client.

- bytes: the content length of the document transferred.

An example of an (artificial) access log entry is:

10023 - - [10/Jun/1998:00:00:01 +0200] “Get /index.html Http/1.0” 2001000.

From the analysis of the overall workload the total number of unique IP addresses that accessed the web site was found to be $2,770,108$. This number can only give a rough 
Stochastic User Behaviour Modelling and Network Simulation for Resource

Management in Cooperating Mobile Telecommunications and Broadcast Networks

estimate of the total number of users due to the presence of proxy caches and shared workstations that hide some of the unique clients and because of the use of Dynamic Host Configuration Protocol, which inflates the number of unique clients. Nevertheless, it is possible to think of this number as the total population accessing the Web site. General statistics regarding the overall set of data are listed in Table 1.

Table 1 Breakdown of HTTP Resource Methods and Response Codes [22]

\begin{tabular}{|l|l|l|}
\hline Method & \% of Requests & \% of Content Data Transferred \\
\hline GET & 99.88 & 99.62 \\
\hline HEAD & 0.10 & 0.30 \\
\hline POST & 0.02 & 0.08 \\
\hline Response Code & \% of Requests & \% of Content Data Transferred \\
\hline 200 (Successful) & 80.52 & 97.86 \\
\hline 304 (Not Modified) & 18.75 & 0.00 \\
\hline Other & 0.73 & 2.14 \\
\hline
\end{tabular}

It can be seen from the data how the 'GET' requests account for almost all the requests for the World Cup site. This is not surprising since the primary purpose of this site was to provide information to people. Also, it can be noticed that the 304 Server Response Codes occupy a quite high percentage of the total requests. It is noted that while the 200 Server Response Code will be returned from the server if the file requested by the client is available for transfer, the 304 code will be returned if the request header included an "if modified since" parameter and the file has not changed since that date. If the file has changed its content since that date and the file is available for transfer a 200 code will be returned. Therefore, 304 coded responses correspond to users refreshing Web pages and traffic analysis of such responses is useful in modelling user behaviour for very popular services such as real time football match scoring as the most passionate and interested users will refresh their page frequently so as not to miss any occurring event. The total number of such users can be used to estimate the percentage of UEs that would subscribe to the "Goal Alert and Multi-view Football" service, since in the UMTS/DVB-T network the action corresponding to refreshing an Internet page, to be updated about the latest events, is to subscribe and consume the Goal Alert and Multiview Football service.

\section{Service Models in the Football Match Scenario}

User behaviour modelling in the Football Match scenario involves a detailed time scale analysis of the Internet data transfer because it shows significant variations in the number of users connected, number of requests, etc., over time so that the average statistics over the entire three-months duration of the data cannot give the right parameters needed for modelling the number of subscriptions during a single football match. For this reason a 


\section{A. Centonza, G.A. Taylor, T. Itagaki, T.J. Owens, J. Cosmas and Y.-H. Song}

Web site access trace corresponding to one of the most popular matches of the tournament (Argentina vs. England) has been chosen for a deeper analysis using MATLAB software [23]. The total number of IP addresses accessing the web site during this match was 60,000, while the total number of IP addresses receiving 304 response codes was 43,300. Other statistics regarding the data trace during this match are listed in Table 2.

Table 2. Breakdown of Resource Methods and Server Response Codes for the match Argentina vs. England.

\begin{tabular}{|l|l|l|}
\hline Method & \% of Requests & $\begin{array}{l}\text { \% of Content Data } \\
\text { Transferred }\end{array}$ \\
\hline GET & 99.94 & 99.82 \\
\hline HEAD & 0.06 & 0.18 \\
\hline POST & 0.00 & 0.00 \\
\hline Response Code & \% of Requests & $\begin{array}{l}\text { \% of Content Data } \\
\text { Transferred }\end{array}$ \\
\hline 200 (Successful) & 64.68 & 98.00 \\
\hline 304 (Not Modified) & 35.11 & 0.00 \\
\hline Other & 0.21 & 2.00 \\
\hline
\end{tabular}

It can be seen that the percentage of 304 response codes given in Table 2 is higher than that given in Table 1 the percentages being approximately $35 \%$ and $20 \%$ respectively. This result supports the assumption that the users receiving 304 response codes are those using the real time scoring service.

\subsection{Multiview Football Service model}

It is possible at this stage to forecast what the user behaviour will be for the Multiview Football service. In fact, if this service is announced only once at the beginning of the match the total number of subscriptions will be proportional to the total number of hosts receiving 304 response codes.

$$
\begin{gathered}
\text { Multiview Football } \\
\text { Subscribers }
\end{gathered}=Q * \begin{aligned}
& \text { Number of Hosts } \\
& \text { receiving } 304 \text { codes }
\end{aligned}
$$

where the constant $Q$ depends on the penetration of the type of technology used, UMTS/DVB-T network, and on the tariff fixed for the service. This is due to the fact that the Multiview Football service is dedicated to the most passionate users, whose "internet identities" are the hosts receiving 304 response codes. In the scenario analysed in this paper a penetration of $10 \%$ is assumed. The constant $Q$ can be calculated as

$$
Q=\frac{\text { Total Number of UEs }}{\text { Number of Hosts }} \text { Total } * C
$$

where $C$ is the factor that takes into account the tariff adopted. At this stage the tariff is not considered, therefore, $C$ could be set arbitrarily to $1, Q$ would have the value 0.1 , and 
Stochastic User Behaviour Modelling and Network Simulation for Resource

Management in Cooperating Mobile Telecommunications and Broadcast Networks

the total number of subscribers for the Multi-view Football service would be 4,330. This total number of users could subscribe to the service within an interval of time between five and ten minutes. In the CISMUNDUS project a period of ten minutes was set, as the maximum length of time the server would wait before streaming the service. This waiting time was set in order to be able to register the number of service subscriptions received before the start of service delivery and decide whether the number of subscribers was high enough to deliver the service via DVB-T. Such a number of users requesting a live streaming video would be very difficult to handle via the UMTS network because of the resource demanding service they want to use. Therefore, if severe losses in revenue and a decrease in user satisfaction due to disconnections and rejections of subscriptions are to be avoided the Multi-view Football service would have to be either suppressed or delivered through a supporting network such as DVB-T.

\subsection{Match Program and Goal Alert Service Models}

For the user behaviour modelling of the "Match Program" and "Goal Alert" services, the model cannot be based only on the total number of hosts connecting to the Web site during the whole match. The Goal Alert service is announced only when an event occurs. Hence, only those users interested in the service at that particular instant of time will access it. For the Match Program service there is the possibility of deploying this service via the UMTS network and announcing the service only when an event occurs. In both cases an analysis of the data on a smaller time scale is required in order to forecast the number of subscriptions per service at a particular instant of time. The analysis of this data leads to the design of user behaviour models based on Poisson processes because, as has been widely reported in literature e.g. [24], the frequency with which a person decides to use the network for a specific task is well described using Poisson processes.

The Argentina vs. England data trace was segmented, into intervals of five minutes each and for each interval statistics are reported in Figures 1 and 2 for the number of hosts receiving 304 codes, the total number of hosts, and the percentages of 304 and 200 codes. Figure 1 shows how the number of hosts receiving 304 codes, together with the total number of hosts connected increased towards the beginning of the penalty kicks. In Figure 2 a very interesting phenomenon can be observed, as we get closer to the most popular event of the match, the penalty kicks, the percentage of 304 codes increases while the percentage of 200 codes decreases. This gives further support for the assumption that the 304 codes represent the most interested and watchful users. 
A. Centonza, G.A. Taylor, T. Itagaki, T.J. Owens, J. Cosmas and Y.-H. Song

Figure 1 Total number of Hosts and number of host receiving 304 response codes, each value plotted represents the average over a five minutes interval

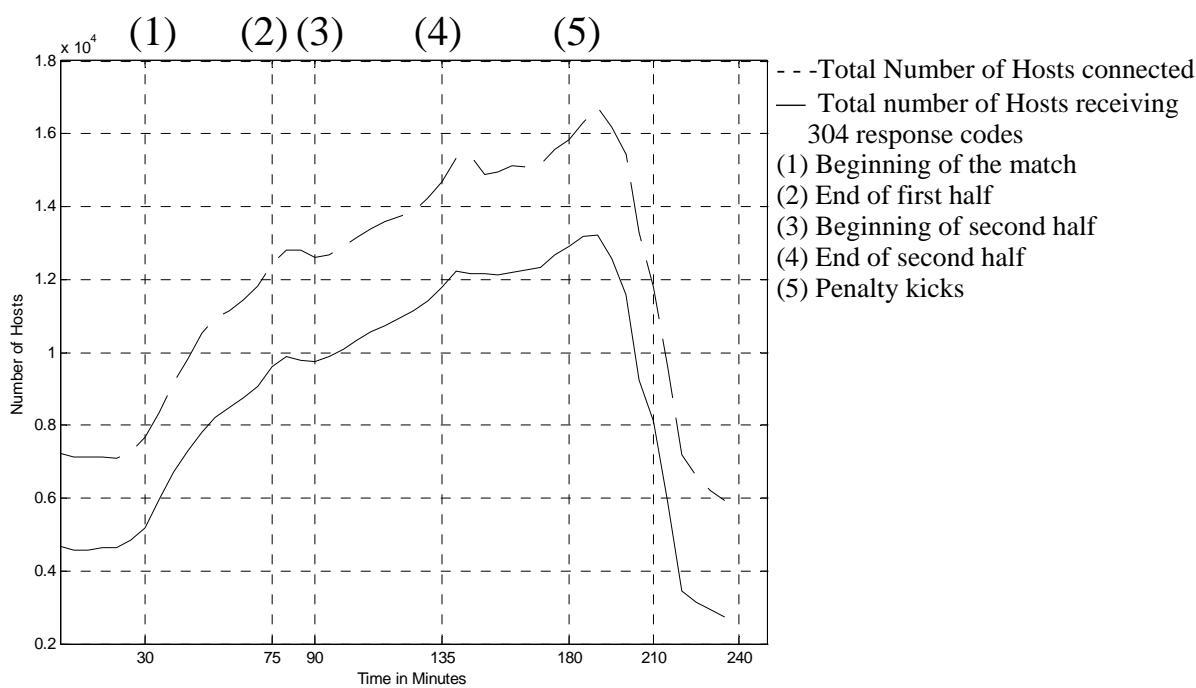

Figure 2 Percentage of 200 and 304 response codes, each value plotted represents the average over a five minutes interval.
(1)
(2) (3)
(4)
(5)

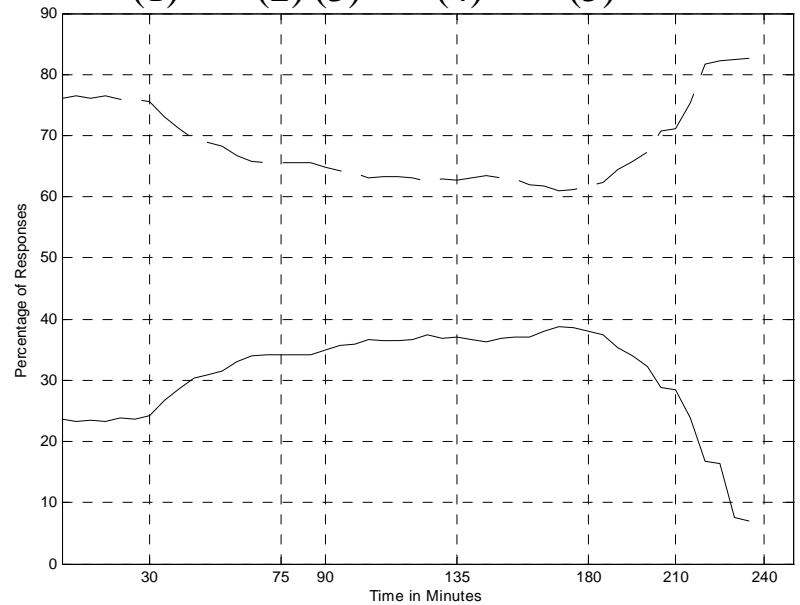

- -Percentage of 200 Response codes

- Percentage of 304 Response codes

(1) Beginning of the match

(2) End of first half

(3) Beginning of second half

(4) End of second half

(5) Penalty kicks 
Stochastic User Behaviour Modelling and Network Simulation for Resource

Management in Cooperating Mobile Telecommunications and Broadcast Networks

\subsubsection{Match Program Model}

From the results shown in Figures 1 and 2 a forecast of the number of subscribers for the Match Program service can be obtained supposing this service is deployed through UMTS and therefore that subscriptions could occur at any time during the match. In this case a non-homogeneous Poisson process determines the number of subscribers where the value of the parameter $\lambda$ for the process is assumed to be proportional to the total number of hosts connected to the site; tariff influence is not considered. This assumption comes from the fact that the Match Program is a service providing general information about the match users are watching with links to Web pages in which other matches of the premier league are described. Therefore, the type of users interested in consuming this service will be any of the users in the stadium carrying a UE, and not only the users calculated from the number of hosts receiving 304 codes.

The parameter $\lambda$ for the non-homogeneous Poisson process is determined for each five minutes interval of time and its value is calculated as follows

$$
\begin{aligned}
& \text { Match Program }=\underline{\text { Total Number of UEs } * f * \quad \text { Total Number of Hosts }} \\
& \text { Average }=\frac{\text { Total Number of Hosts }}{\text { Total }} \text { Connected During the actual } \\
& \text { Subscribers }(\lambda) \quad 5 \text { minutes interval }
\end{aligned}
$$

For user behaviour modelling of this service it has been taken into consideration that during the match users will be busy following its events and therefore it will be less probable that they will consume the Match Program service. Therefore, the number of subscriptions is forecast to have more spikes and sinks, than in the World Cup traces, with very high fluctuations between the play-time and the intervals. In Equation (5) this phenomenon is taken into account through the parameter $f$. This parameter shapes the curve of number of subscriptions against time so that the increases in subscriptions follow an exponential trend and the decreases follow a hyperbolic trend. This choice has been taken after analysing the 1998 World Cup Web Site data set on a very large time scale and noticing that the rises and falls in the total number of hosts for each match played followed these trends. In addition, due to the different usage time of the service in case of wireless and Internet users, assumptions must be made on when the peak demand occurs if the service is deployed on the hybrid UMTS/DVB-T platform. Indeed, a demand peak such as that shown in Figure 1 occurring at the end of the first half would be very unlikely to happen in the UMTS/DVB-T scenario because the users would be watching the match instead of enjoying the services. Such peak would be more likely to occur during the interval between first and second half, when users could be "playing" with their handsets. For this reason the three peaks visible in Figure 1 occurring at the end of the first half, end of the second half and at the beginning of the penalty kicks have been used to estimate the peak load imposed on the UMTS/DVB-T network assumed to occur before the start, during the first half and after the end of the football match. It is worth noting that during these peak times the parameter $f$ equals one, therefore the forecast of the peak number of subscriptions relies entirely on the data collected from the 
Web Site. Figure 3 shows the simulated number of subscribers for the Match Program service. It can be observed from this figure how the peaks are reached when the match is not playing, while during the match the number of users requesting the service goes down to values resembling the number of subscribers before the start of the match. If the service is announced before the start of the match this result could be used to forecast the number of subscribers during the interval and at the end of the match and give an idea of when it is most opportune to announce the service in order to receive the maximum number of subscriptions useful to predict the delivery mechanism to be employed.

Figure 3 Simulation results representing the number of subscribers for the Match Program Service.

(1) (2) (3)

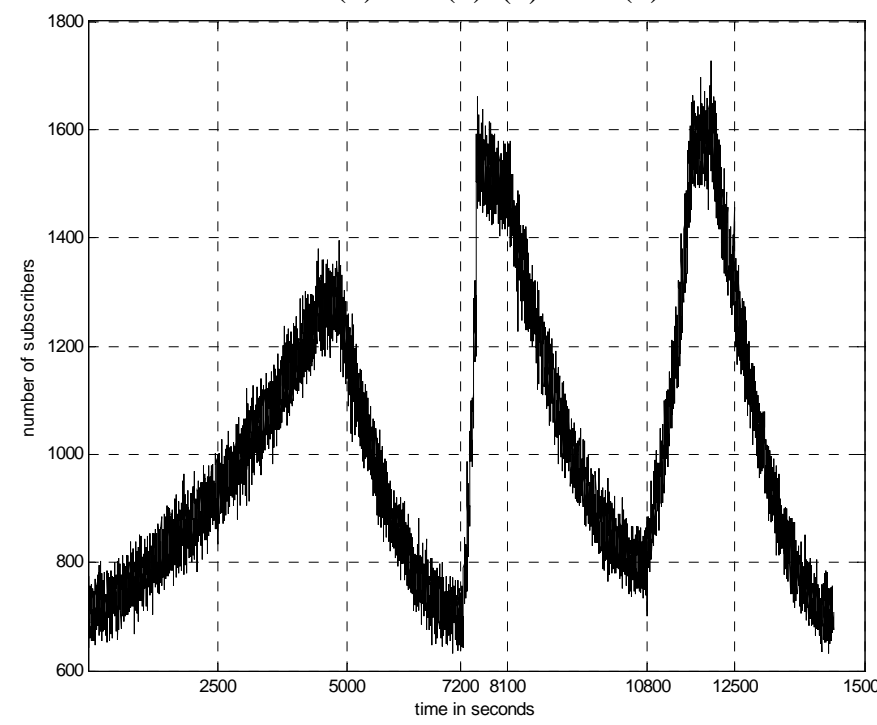

(1) Beginning of the match

(2) End of first half

(3) Beginning of second half

(4) End of the match

\subsubsection{Goal Alert Model}

For the "Goal Alert" service a different approach is needed because it is announced only when an event occurs in one of the other matches of the premier league. Therefore, the announcement is not a scheduled event, but it is a spontaneous event to which a distribution has to be fitted. For this purpose a stochastic model describing the occurring of events in seven football matches played at the same time has been used. The model is based on historical data collected from five sets of seven matches each played in the 2002-2003 Italian premier league and it is based on a Poisson process. Events are classified depending on their popularity. Three levels of popularity have been created: high, medium and low. For example, a goal scored in a very important match of the premier league will fall into the high popularity class, while the booking of a player in a 
Stochastic User Behaviour Modelling and Network Simulation for Resource

Management in Cooperating Mobile Telecommunications and Broadcast Networks

less important match will be classified as a low popularity event. The output of the stochastic model is shown in Figure 4. The result shown in Figure 4 is used to determine when subscriptions for this service will occur. In order to determine the number of users subscribing at each announcement, three average values of subscriptions have been assigned, one for each class of events. These values have been calculated as the maximum number of hosts receiving 304 response codes in a five minutes interval taken from three matches of the France'98 World Cup. Each match corresponds to a popularity class: Argentina vs. England (high popularity); Netherlands vs. Yugoslavia (medium popularity) and Brazil vs. Morocco (low popularity). The average values of subscribers for each class are listed in Table 3. A Poisson model with average values equal to the values listed in Table 3 determines the predicted number of subscriptions occurring for each event. The final result for the number of subscriptions predicted at each announcement of the Goal alert service is shown in Figure 5.

By comparing Figure 4 and Figure 5 it is possible to see how when the events occur the number of predicted subscriptions occurs. From the results shown in Figure 5 it can be deduced that the deployment of the video clips for the Goal Alert service should occur through DVB-T, because as demonstrated in the next section UMTS networks could not cope with such a high service demand.

Figure 4 Output of the stochastic model used for calculation of announcement times.

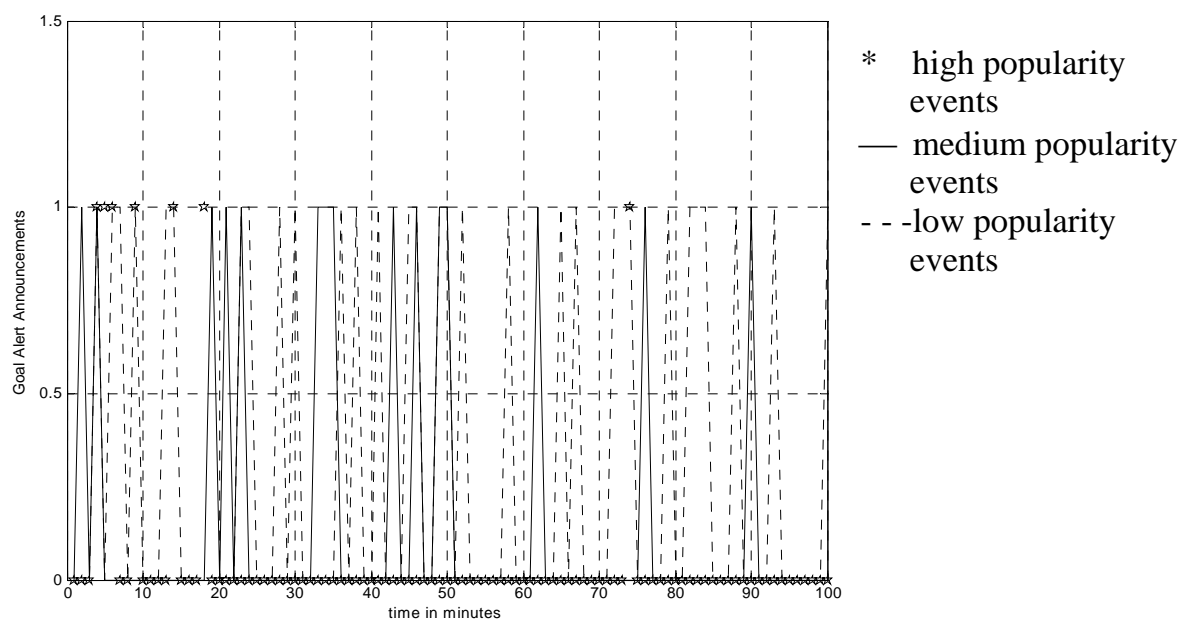

Table 3. Average value of subscriptions per popularity class for the Goal Alert service.

\begin{tabular}{|c|c|c|c|}
\hline & $\begin{array}{c}\text { High } \\
\text { Popularity }\end{array}$ & $\begin{array}{c}\text { Medium } \\
\text { Popularity }\end{array}$ & $\begin{array}{c}\text { Low } \\
\text { Popularity }\end{array}$ \\
\hline $\begin{array}{c}\text { Average Number of } \\
\text { Subscriptions }\end{array}$ & 1320 & 869 & 445 \\
\hline
\end{tabular}


Figure 5 Simulated number of Subscribers for Goal Alert service over 100 minutes.

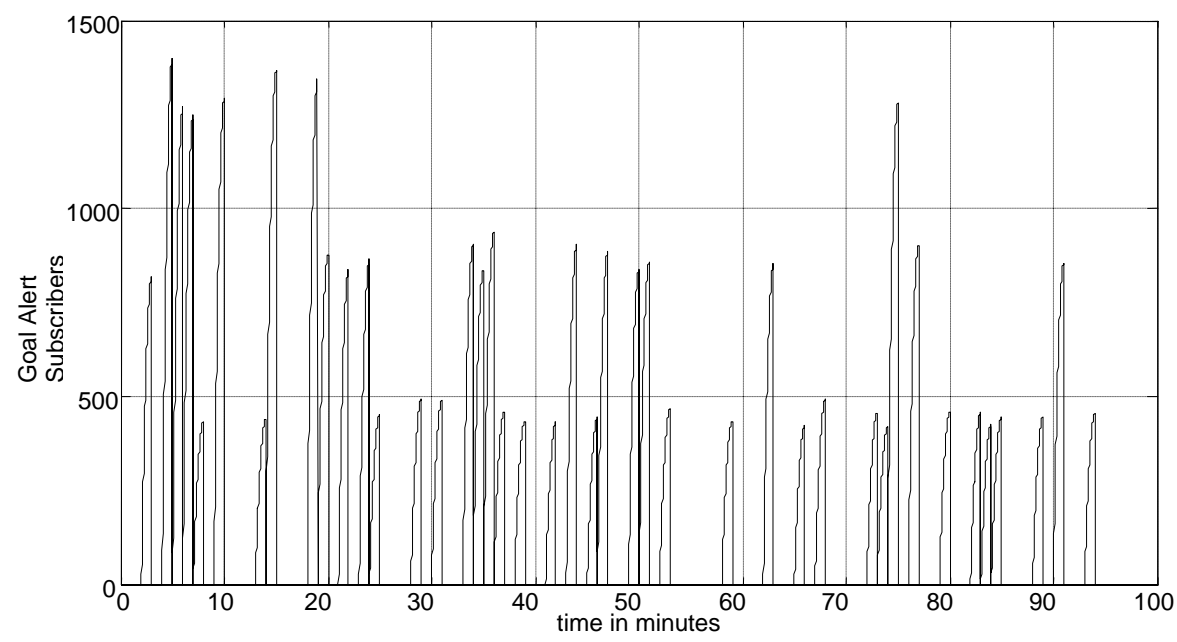

\section{Network Simulations and Results}

The results reported in the previous section give a forecast of what could be the utilisation of the services listed in Section 3. In this section it is shown how the UMTS network would react to such traffic. Once this is established, it will be possible to state whether the deployment of these services has to happen via UMTS or via a supporting network such as DVB-T.

Of the three services presented only one will be simulated: the Goal Alert service. The reason for this choice is that this is the service for which the minimum number of subscriptions is forecast and therefore more suitable for testing on a telecommunications network such as UMTS. Moreover, this is the service with the lowest QoS requirements and the least demanding in terms of network resources. If in this case it is shown that the performance of the UMTS network is not satisfactory, it will be straightforward to conclude that the other services cannot be deployed via UMTS alone.

To perform such a simulation a UMTS network model has been developed using the OPNET simulation software. The model was developed in several steps and its capacity has been simulated under different conditions. The coverage of the football stadium has been simulated for different solutions such as: single cell, single cell-three sectors, two cells, two cells-three sectors and four cells-three sectors, where "three sectors" means that instead of standard base stations with isotropic antennas, base stations using sectors of 120 degrees have been used. In order to improve the number of users admitted for service consumption several parameters were modified as the number of UEs was increased. Some of these modifications were: The RLC Timer Discard (elapsed time 
Stochastic User Behaviour Modelling and Network Simulation for Resource

Management in Cooperating Mobile Telecommunications and Broadcast Networks

before a Segment Data Unit (SDU) is discarded) was increased from an initial value of 1,000 ms up to 7,500 ms; TCP Maximum Connect Attempts were increased from three to five; TCP Maximum Data Retransmission Attempts were increased from four to six; the interval of time during which each user will try to set up a connection was increased from 60 s to 80 s. The remaining parameter settings used in the model and kept unchanged throughout the simulations are listed in Table 4, while a representation of the model is given in Figure 6.

Table 4. UMTS Simulation Model Settings

\begin{tabular}{|c|c|}
\hline Orthogonality Factor & 0.9 \\
\hline Other Cell/Own Cell Interference & 0.56 \\
\hline Thermal Noise Power Spectral Density & $-174 \mathrm{dBm} / \mathrm{Hz}$ \\
\hline Node B Max Transmission Power & $20 \mathrm{~W}$ \\
\hline UE Max Transmission Power & $0.5 \mathrm{~W}$ \\
\hline Max Downlink Bit Rate & $64 \mathrm{Kbps}$ \\
\hline Max Uplink Bit Rate & $32 \mathrm{Kbps}$ \\
\hline
\end{tabular}

Figure 6 UMTS simulation model obtained using OPNET simulation software.

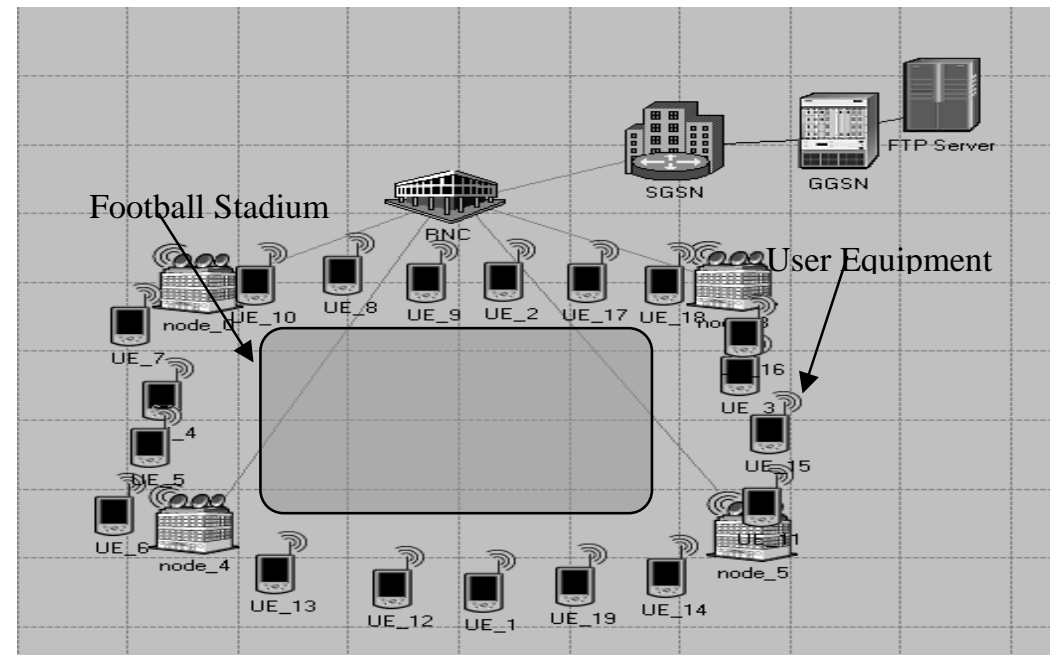

The model is used to simulate the download of a $420 \mathrm{~KB}$ file from a FTP server. This represents the download of a 10 seconds video clip in MPEG4 format in the Goal Alert scenario. The QoS chosen for this type of service is Background due to the fact that this 
is the most commonly used QoS class for FTP services. Within an interval of time from 60 s to 80 s, all the users will try to set up a connection and start the download. In order to test the capacity of the network, several situations of overload are simulated where users will be either rejected by the admission control algorithm during the connection set-up procedure or be disconnected during the communication. The overall number of "not served" subscribers does not change linearly with the total number of UEs due to the increase in the interference level, caused by the increase in the number of UEs, see Equation (4). Therefore, the total number of rejections will increase faster than linearly with the total number of users. Also, the QoS characteristics will deteriorate as the total number of users increases. A list of the results obtained from the simulations performed is given in Table 5.

Table 5. Simulation Results

\begin{tabular}{|c|c|c|c|}
\hline Scenario Statistics & Users Rejected & $\begin{array}{c}\text { Average connection } \\
\text { time }\end{array}$ & $\begin{array}{c}\text { Average TCP } \\
\text { Retransmission Count }\end{array}$ \\
\hline Single Cell / 17 Users & 4 & $66 \mathrm{~s}$ & 7 \\
\hline $\begin{array}{c}\text { Single Cell-Three Sectors / } \\
\text { 30 Users }\end{array}$ & 0 & $100 \mathrm{~s}$ & 50 \\
\hline Two Cells 24 Users & 0 & $120 \mathrm{~s}$ & 36 \\
\hline $\begin{array}{c}\text { Two Cells-Three Sectors / } \\
\text { 46 Users }\end{array}$ & 0 & $126 \mathrm{~s}$ & 125 \\
\hline $\begin{array}{c}\text { Two Cells-Three Sectors / } \\
54 \text { Users }\end{array}$ & 4 & $142 \mathrm{~s}$ & 95 \\
\hline $\begin{array}{c}\text { Four Cells-Three Sectors / } \\
68 \text { Users }\end{array}$ & 0 & $112 \mathrm{~s}$ & 106 \\
\hline $\begin{array}{c}\text { Four Cells-Three Sectors / } \\
78 \text { Users }\end{array}$ & 8 & $117 \mathrm{~s}$ & 114 \\
\hline $\begin{array}{c}\text { Four Cells-Three Sectors / } \\
\text { 105 Users }\end{array}$ & 14 & $120 \mathrm{~s}$ & 136 \\
\hline $\begin{array}{c}\text { Four Cells-Three Sectors / } \\
\text { 145 Users }\end{array}$ & 25 & $138 \mathrm{~s}$ & 106 \\
\hline
\end{tabular}

The first thing that can be noticed by looking at the results in Table 5 is that the three sectors base stations perform much better than the isotropic ones. This is not surprising given that for average interference conditions each sector generally provides a capacity increase of $90 \%$ [17]. The second phenomenon that can be observed by looking at Table 5 is the loss of revenue an operator would face if the number of users became much higher than the network could handle. It can be seen that although the UMTS network performs well in cases of limited numbers of service subscribers, an increase in rejections is experienced for scenarios with more than 78 users. In the Four Cells-Three sectors case with 145 users, although the user subscription time interval has been increased from 60 s to 80 s, the total number of users rejected goes up to over $17 \%$ of the total number of UEs. This is the reason why the more heavily loaded scenarios have not been simulated, assuming that the maximum percentage of rejections allowed is fixed by the network operator at $15 \%$ of the overall number of subscribing users it is possible from the scenarios considered to estimate the capacity offered by the UMTS network. The last 
Stochastic User Behaviour Modelling and Network Simulation for Resource

Management in Cooperating Mobile Telecommunications and Broadcast Networks

two characteristics highlighted by the results are the increases in the Average Connection Time and the Average TCP Retransmission Count. These parameters can be described as follows: the Average Connection Time represents the average interval of time measured from the time a client application sends a request to the server to the time it receives a response packet, while the Average TCP Retransmission count represents the average number of total TCP data retransmissions in the network. The first of these two parameters gives an estimate of the average time a user has to wait in order to start downloading the file. This time does not represent the total time the user should wait before being able to watch the video clip. If this figure has to be found, the Average Connection Time should be added to the download time experienced by the user. Due to the fact that the download time can be highly variable from user to user the total time has not been reported. Nevertheless, the Average Connection Time provides important information about the QoS provided in terms of delay, as it is one of the most influential parameters for user satisfaction. The second parameter gives information about how well the network is performing in terms of users admission and data transmission. In fact, if at a certain instant of time the maximum number of UEs connected is reached, every further UE trying to set up a connection will be rejected. However, the rejected UE will try to connect again after a certain interval of time by re-transmitting the connection set up request and if rejected again will keep on trying until the TCP Maximum Connect Attempts value is reached. In this case the UE will send a connection abort message and will not try to connect again. This phenomenon will cause an increase in the number of TCP retransmissions experienced by the network that will be reflected in an increase in the Average Connection Time. On the other hand, if a UE is admitted for the connection set up and the interference level or the network congestion level or both are very high some of the data packets will be lost during transmission or corrupted. In this case, the data will need to be retransmitted causing an increase in the Average TCP Retransmission Count. A high value of the Average TCP Retransmission Count is therefore synonymous with poor data transmission and consequently with QoS deterioration.

If this service were delivered via DVB-T, any limit in the number of users would be determined by the uplink capacity of the UMTS network. In fact, in the hybrid UMTS/DVB-T platform developed in CISMUNDUS project the signalling between UEs and core network always happens via the uplink channels of the UMTS network. Therefore, in order to give an estimate of the maximum uplink capacity in this scenario the statistics necessary to compute Equation (3) were collected and the number of UEs for which the pole capacity is reached calculated. In the Four Cells-Three Sectors / 145 Users scenario, the average $E_{b} / N_{o}$ for the uplink signalling channels is $4.5 \mathrm{~dB}$, while the maximum bit rate is 500 bps. Hence, according to Equation (3), the uplink pole capacity in these conditions would be reached with a total number of users per cell/sector of 1,736. This value is an overestimation of the real possible number of users allowable, due to the fact that in Equation (3) the increase in $E_{b} / N_{o}$ caused by the higher number of mobiles served has not been taken into account. However, such a high number of possible users 
suggests that even after an increase in the interference level the use of the DVB-T network would make possible the provisioning of the service to all the forecast subscribers for all the services analysed avoiding rejections. Moreover, deployment via DVB-T would be even more advantageous in case of services such as Multiview Football. This service consists of a streamed video and as such it would be delivered using UDP transport protocol and RLC unacknowledged mode instead of TCP and eventually RLC acknowledged mode. This implies the use of the uplink UMTS channel for service subscription only avoiding the signalling due to acknowledgments and therefore increasing the overall capacity of the platform.

\section{Conclusions}

In this paper traffic modelling of multimedia services planned for deployment on an UMTS/DVB-T network has been undertaken. User behaviour models for predicting the demand for the services have been developed with an emphasis on forecasting the maximum number of subscribers for each service. The contribution of this paper is the development of stochastic models for predicting user behaviour for specified prospective service scenarios based of the number of hosts receiving 200 and 304 response codes in an Internet scenario, providing services similar to those specified. The approach presented could be applied to a number of other service scenarios provided that corresponding Internet service scenarios can be found. Some of the models developed have been used in OPNET simulations of one of the prospective services considered. Simulation results have been reported to provide telecommunication network operators with useful information on resource management and traffic routing in a telecommunications network that is supported by a broadcast network. The results show that in cases of severe overload the delivery of the prospective services via UMTS would be uneconomical and would lower the user satisfaction. An estimate of the maximum number of subscribers in the case of service deployment supported by a DVB-T network shows how the use of DVB-T can guarantee a much higher capacity and revenue. The simulations also show how UMTS is unable to cope with a bursty number of subscriptions justifying the broadcasting of high demand traffic to relieve congestion.

\section{Acknowledgements}

The authors would like to acknowledge Mr. Martin Arlitt for useful suggestions provided on the statistical analysis of data.

\section{References}

[1] Berg, M., Butterfield, S., Cosmas, J., Casagranda, P., Garrec, D., Guiraudou, M., Martinez, G., Launay, E., Mazieres, B., Milanesio, D., (2003) 'CISMUNDUS: Convergence of Digital 
Stochastic User Behaviour Modelling and Network Simulation for Resource Management in Cooperating Mobile Telecommunications and Broadcast Networks

Broadcast and Mobile Telecommunications' Proceedings of the International Broadcasting Convention, 12-16 September.

[2] Mylonakis, J., (2004) 'Can mobile services facilitate commerce? Findings from the Greek telecommunications market’, International Journal of Mobile Communications, Vol. 2, No. 2, pp. 188 - 198.

[3] Sigurdson, J., Ericsson, P., (2003), 'New services in 3G - new business models for streaming and video', International Journal of Mobile Communications, Vol. 1, No 1/2, pp. 15-34.

[4] Jorgusesky, L., Fledderus, E., Farserotu, J., Prasad, R., (2001) 'Radio Resource Allocation in Third-Generation Mobile Communication Systems’ Communication Magazine, IEEE, Vol. 39, Issue 2, pp. 117-123.

[5] Seong-Jun, O., Danlu, Z., Wasserman, K.M., (2003) 'Optimal resource allocation in multiservice CDMA networks' Transactions on Wireless Communications, IEEE, Vol. 2, Issue 4, pp. 811-821.

[6] Almeida, S.; Queijo, J., Correia, L.M., (1999), 'Spatial and temporal traffic distribution models for GSM'. VTC 1999 - Fall. IEEE VTS 50 th, $19-22$ September, Vol. 1, 19-22, pp. 131135.

[7] Darwood, P., Oppermann, I., Jakas, S., Linton, W., (2000) 'Mobile network traffic forecasting. Vehicular Technology Conference’, IEEE, VTS-Fall VTC 2000. 52nd, 24-28 September, Vol. 6, pp. 2932-2936.

[8] Ribeiro,L.Z., DaSilva, L.A., (2002) 'A framework for the dimensioning of broadband mobile networks supporting wireless Internet services’, Wireless Communications, IEEE, Vol. 9, Issue 3, pp. 6-13.

[9] Trangia, P., Staehle, D., Leibnitz, K., (2001) 'Source Traffic Modelling of Wireless Applications'. International Journal of Electronics and Communications, Vol. 55, Issue 1, pp. 27-36.

[10] Klemm, A., Lindemann, C., Lohmann, M., (2001) 'Traffic Modeling and Characterization for UMTS Networks'. Global Telecommunication Conference, GLOBECOM’01, San Antonio TX, 25-29 November, Vol 3, pp. 1741-1746.

[11] Janevski, T., (2003) Traffic Analysis and Design of Wireless IP Networks, Artech House.

[12] 3GPP, (1998) 'Universal Mobile Telecommunication System (UMTS), Selection Procedures for the Choice of Radio Transmission technologies of the UMTS', 3GPP TR 101-112, v. 3.2.0, April.

[13] Lim, B., Zheng, H., (2004) 'A day in the life of Jini: a peek at service-oriented architecture for internet appliances', International Journal of Mobile Communications, Vol. 2, No 2, pp. 199-216.

[14] OPNET (2004), 'Making Networks and Applications Perform', $11^{\text {th }}$ May, http://www.opnet.com

[15] Banitsas, K.A., Song, Y.-H., Owens, T. J., (2004) 'OFDM over IEEE 802.11b hardware for telemedical applications', International Journal of Mobile Communications, Vol. 2, No 3, pp.310-327.

[16] Korhonen, J., (2001) Introduction to 3G Mobiles Communications, Artech House.

[17] Holma, H., Toskala, A., (2001) WCDMA for UMTS, John Wiley and Sons.

[18] Brunel University (2004), 'CISMUNDUS Home Page' $10^{\text {th }} \quad$ February, http://www.brunel.ac.uk/project/Cismundus 


\title{
A. Centonza, G.A. Taylor, T. Itagaki, T.J. Owens, J. Cosmas and Y.-H. Song
}

[19] Cosmas, J., Elgohari, L., Haque, M., Itagaki, T., Krishnapillai, K., Lucas, A., Zheng, L. (2003) 'Providing Fast Prototypes of Services and Automated Generation of Service Applications for Converged Broadcast and Cellular Networks' Proceedings of the HyWin Conference, Turin, 2nd December.

[20] Vrechopoulos, A., Constantiou, I., Sideris, I., Doukidis, G., Mylonopoulos, N., (2003) 'The critical role of consumer behaviour research in mobile commerce', International Journal of Mobile Communications, Vol. 1, No. 3, pp.239 - 304.

[21] The Internet Traffic Archive, ACM/SIGCOMM (2004), 'Traces in the Internet Traffic Archive' $10^{\text {th }}$ February, http://ita.ee.lbl.gov/html/traces.html

[22] Arlitt, M. and Jin, T., (1999) 'Workload Characterization of the 1998 World Cup Web Site' HP Labs Technical Reports: HPL-1999-35R1 (991006).

[23] The MathWorks, (2004), 'Accelerating the Pace of Engineering and Science', 3rd of August, http://www.mathworks.com

[24] Paxson, V. and Floyd, S., (1995) 'Wide Area Traffic: the Failure of Poisson Models'. Transactions on Networking, IEEE/ATM, Vol.3, Issue 3, pp. 266-244.

\begin{abstract}
The rapid growing in demand for mobile multimedia services is pushing mobile telecommunications operators towards cooperation with more capable networks such as broadcast. In this paper user behaviour analysis and network simulations are performed for a hybrid UMTS/DVB-T network scenario. The results provide network operators with useful information and ideas about traffic delivery and network management.
\end{abstract}

